\title{
First estimate of wind fields in the Jupiter polar regions from JIRAM- Juno images
}

D. Grassi ${ }^{1}$, A. Adriani ${ }^{1}$, M.L. Moriconi ${ }^{2}$, A. Mura ${ }^{1}$, F. Tabataba-Vakili ${ }^{3}$, A. Ingersoll ${ }^{4}$, G. Orton ${ }^{3}$, C. Hansen $^{5}$, F. Altieri ${ }^{1}$, G. Filacchione ${ }^{1}$, G. Sindoni ${ }^{1}$, B.M. Dinelli ${ }^{6}$, F. Fabiano ${ }^{6}$, S. J Bolton ${ }^{7}$, S. Levin $^{3}$, S.K. Atreya ${ }^{8}$, J.I. Lunine ${ }^{9}$, T. Momary ${ }^{3}$, F. Tosi ${ }^{1}$, A. Migliorini ${ }^{1}$, G.Piccioni ${ }^{1}$, R. Noschese ${ }^{1}$, A. Cicchetti ${ }^{1}$, C. Plainaki ${ }^{10}$, A. Olivieri ${ }^{11}$, D. Turrini ${ }^{1}$, S. Stefani ${ }^{1}$, R. Sordini ${ }^{1}$, M. Amoroso ${ }^{11}$

1. Istituto di Astrofisica e Planetologia Spaziali - Istituto Nazionale di Astrofisica, Rome, Italy

2. Istituto di Scienze Atmosferiche e del Clima, Consiglio Nazionale delle Ricerche, Sede di Roma, Italy

3. Jet Propulsion Laboratory, California Institute of Technology, Pasadena, California, USA

4. California Institute of Technology, Pasadena, California, USA

5. Planetary Science Institute, Tucson, Arizona, USA

6. Istituto di Scienze Atmosferiche e del Clima, Consiglio Nazionale delle Ricerche, Sede di Bologna, Italy

7. Southwest Research Institute, San Antonio, Texas, USA

8. University of Michigan, Ann Arbor, Michigan, USA

This is the author manuscript accepted for publication and has undergone full peer review but has not been through the copyediting, typesetting, pagination and proofreading process, which may lead to differences between this version and the Version of Record. Please cite this article as doi: 10.1029/2018JE005555 
9. Cornell University, Ithaca, New York, USA

10. Agenzia Spaziale Italiana, Sede di Roma, Italy

11. Agenzia Spaziale Italiana, Sede di Matera, Italy

* Corresponding author: davide.grassi@iaps.inaf.it

\begin{abstract}
We present wind speeds at the $\sim 1$ bar level at both Jovian polar regions inferred from the $5-\mu \mathrm{m}$ infrared images acquired by the Jupiter InfraRed Auroral Mapper (JIRAM) instrument on the NASA Juno spacecraft during its fourth periapsis (February $2^{\text {nd }} 2017$ ).

We adopted the criterion of minimum mean absolute distortion (Gonzalez and Woods, 2008) to quantify the motion of cloud features between pairs of images.

The associated random error on speed estimates is $12 \mathrm{~m} / \mathrm{s}$ in the Northern polar region and $9.8 \mathrm{~m} / \mathrm{s}$ at the South.

Assuming that polar cyclones described by Adriani et al. (2018) are in rigid motion with respect to System III, tangential speeds in the interior of the vortices increase linearly with distance from the center. The annulus of maximum speed for the main circumpolar cyclones is located at approximatively $1000 \mathrm{~km}$ from their centers, with peak cyclonic speeds typically between 80 and $110 \mathrm{~m} / \mathrm{s}$, and $\sim 50 \mathrm{~m} / \mathrm{s}$ in at least two cases. Beyond the annulus of maximum speed, tangential speed decreases inversely with the distance from the center within the Southern Polar Cyclone and somewhat faster within the Northern Polar Cyclone.
\end{abstract}

A few small areas of anticyclonic motions are also identified within both polar regions.

\title{
Keywords
}

Jupiter atmosphere, wind speeds, planetary vortices

\section{Introduction}

The estimate of wind fields from the tracking of cloud features represents a key tool in constraining the dynamical behavior of planetary atmospheres. The case of Jupiter is, by no means, an exception. Global-scale zonal winds profiles have been derived from a variety of datasets: Voyager Imaging Science System [Ingersoll et al., 1981, Limaye, 1986], Hubble Space Telescope [Garcìa-Melendo \& Sánchez-Lavega, 2001], Cassini Imaging Science Subsystem, during the Jupiter flyby of December 2000 [Porco et al., 2003] and ground-based telescopic observations [Hueso et al., 2017]. These studies suggest a long-term stability of the inferred latitudinal profiles of mean zonal winds. On a local scale, wind fields have been derived, among others, for the Great Red Spot [Read et al., 2006, Choi et al., 2007, Asay-Davis et al., 2009] and hot spots and plume areas [Hueso and SánchezLavega, 1998] on the basis of Voyager, Galileo and Hubble Space Telescope data. 
Moving to higher latitudes, a system of circumpolar waves in Jupiter's stratosphere was firstly identified by Sánchez-Lavega et al., [1998] while Vincent et al. [2000] described extensively the motions in Jupiter's stratosphere with description of specific features up to approximatively $75^{\circ}$. More recently, Barrado-Izagirre et al., [2008] focused their attention on circumpolar regions at latitudes below $80^{\circ}$, where longitudinal variations appears to be caused by Rossby waves.

However, very limited information on polar regions beyond the $80^{\circ}$ latitude was available prior to 2016. Ground-based observations are adversely affected by high viewing angles, a factor difficult to mitigate due to the very small axial tilt of the planet. Furthermore, the trajectories of Voyager, Cassini, Galileo and New Horizons spacecraft through or in the Jupiter system remained rather close to Jupiter's equatorial plane, resulting in similar viewing limitations. Pioneer 11 passed over the north pole of Jupiter, but could not measure winds due to limitations in the imaging system and to the very large distance of the observations [Baker et al., 1975, Orton et al., 1981]. On July $4^{\text {th }}$, 2016 the Juno spacecraft entered into a polar orbit around Jupiter, providing the opportunity to acquire the best view of its polar regions achieved so far [Bolton et al., 2017]. Among the Juno instruments, the Jupiter InfraRed Auroral Mapper (JIRAM) [Adriani et al., 2014] is proving particularly useful in studying the upper troposphere [between 5 and 1 bars] at high latitudes. The instrument includes an IR-imager channel equipped with a broad-band filter centered at $4.8 \mu \mathrm{m}$. This wavelength region allows one to cover simultaneously the day and night side of the polar regions, enabling synoptic coverage of large areas not achievable by observations acquired at shorter wavelengths dominated by reflected sunlight. The $4.8-\mu \mathrm{m}$ region hosts an important transparency window of the gaseous absorption spectrum. In absence of clouds, the ultimate source of opacity is represented by the $\mathrm{H}_{2}$ collision-induced absorption and observed thermal radiation emerges from a pressure level calculated to be about 5.5 bars [see discussion in Grassi et al., 2017a and Grassi et al., 2017b]. The absence of clouds above the 5-bar level is, however, a condition very rarely seen: globally, averaged equilibrium thermodynamics models predict several different cloud layers of ammonia, ammonium hydrosulfide and water, extending over several tens of kilometers in altitude [Atreya et al., 1999]. The analysis of Galileo Near Infrared Mapping Spectrometer (NIMS) spectra by Irwin et al., [2001] found that changes in brightness at $5 \mu \mathrm{m}$ - at least at the low and intermediate latitudes considered in their study - are correlated not with variability of the higher ammonia cloud, but rather with opacity variations associated with cloud layers between 1 and 2 bars of pressure. In the subsequent discussion, we will assume that features observed in JIRAM images reside in this particular pressure range.

The overall morphologies of north and south poles of Jupiter as seen by JIRAM and by the JunoCam instruments were described by Adriani et al., [2018]. In the current work we adopt the features nomenclature presented in that paper; a suffix ' $n$ ' or ' $s$ ' is added where needed to distinguish northern and southern features. The north pole hosts a central vortex that rotates counterclockwise: this structure is named NPC (as for 'North Polar Cyclone') in Adriani et al., [2018], and as such is 
labeled in Figure 1a. Given its location in the northern hemisphere it will be considered a cyclone, with a local minimum of pressure at its center. This cyclone shows a low infrared brightness (suggestive of relatively thick cloud coverage), with sizes of turbulent eddies developed down to the smallest scales resolved in the images (about $12 \mathrm{~km}$ ). NCP is surrounded by four additional, equally spaced, cyclones, all of which have centers at latitude $83.3^{\circ}$. Three of these cyclones present internal features (approximately within $1000 \mathrm{~km}$ from respective centers) with turbulent eddies with sizes down to few tens of kilometers (among them, CPCn6 and CPCn2, where CPC stands for 'Circum-Polar Cyclone'), while the fourth (CPCn8 of Adriani et al., [2018], located at $135^{\circ} \mathrm{W}$, not shown in Figure 1a) has a central part with a more 'laminar' aspect, with eddy scales appearing to be limited to at least $100 \mathrm{~km}$. Four other equally spaced cyclones with centers at $82.5^{\circ}$ and spaced $45^{\circ}$ from the first set of four complete the north pole dynamical organization (among them, CPCn3 and CPCn7). The external four cyclones again present a 'laminar' aspect, with an apparent lack of smallscale eddies in their centers. The south pole presents a slightly more complex morphology. A central 'laminar' vortex (SPC in Figure 1b, as for 'South Polar Cyclone') exists there, but its apparent center is more clearly displaced from geographical pole than its northern counterpart. SPC is surrounded by five vortices irregularly spaced and of different appearance. All six main vortices of the south pole rotate in cyclonic motions(clockwise in the southern hemisphere), suggesting low-pressure centers. While all cyclones show turbulent external parts, with eddies developed at a variety of scale lengths, only three vortices (CPCs 2, 5 and 1) have low-brightness, turbulent centers with eddies of the size of few tens of kilometers, while the remaining two (CPCs 6 and 3, the former not shown in Figure 1b) present 'laminar' central parts.

The methods described in Grassi et al. [2017b], albeit developed for the specific conditions of Hot Spots, can be tentatively applied to the JIRAM spectra acquired over the two poles, to obtain a quantitative estimate of aerosol optical thickness at $5 \mu \mathrm{m}\left(\tau_{5 \mu \mathrm{m}}\right)$, in the assumption of a single cloud deck located at about 1 bar. Results indicate that clouds over extended areas in the the NPC present $\tau_{5 \mu \mathrm{m}}$ exceeding 6 , while they remain considerably optical thinner $\left(\tau_{5 \mu \mathrm{m}} 2-3\right)$ in CPCn2 and CPCn6. In the south pole, $\tau_{5 \mu \mathrm{m}}$ above 6 are found in the central parts of CPCs4, 5 and 1 , as well as in the area between CPCs4, 1 and SPC. Most of the SPC, as well as the central part of CPCs3 appears to have $\tau_{5 \mu \mathrm{m}} 2-3$.

Adriani et al. [2018] presented preliminary estimates of tangential speeds of the polar vortices observed by JIRAM. In the current paper, we substantially extend those findings, presenting the wind fields derived over large continuous areas of both polar regions by a fully automated algorithm. We use JIRAM images acquired during the fourth Juno close passage ("perijove") (PJ4) on February $2^{\text {nd }} 2017$, the most recent flyby for which sufficient numbers of image pairs are available for wind speed computations.

\section{Materials}

The JIRAM instrument includes a spectrometer and two image channels operating in different 
spectral regions. In this paper, we will focus our attention solely on one of these channel (the so called M-filter), where images are acquired by integrating the incoming radiance over the 4.54- 5.03 $\mu \mathrm{m}$ range. Images have a size of 432 x 128 pixels. All image pixels are acquired simultaneously, i.e.: the bi-dimensional image is formed directly on the focal plane and not by the stacking of consecutive lines in a push broom scan. The field of view of individual pixels is about $240 \mu \mathrm{rad}$. A complete description of the JIRAM instrumen can be found in [Adriani et al., 2014]

Juno is a spin-stabilized spacecraft and JIRAM acquires one image at each Juno spacecraft rotation ( $2 \mathrm{rpm}$ ). JIRAM pointing has only one degree of freedom, and the pointing can be set only along the maximum circle orthogonal to the spin axis. The Juno spin axis is kept pointed toward the Earth and, therefore, roughly toward the Sun. Moreover, the Juno orbital plane is approximately orthogonal to the spacecraft's spin axis. As a consequence of these constraints, JIRAM images acquired near the close approach are often placed very close to the planet terminator. In most circumstances, JIRAM is operated to acquire a set of consecutive images (a "sequence"), spaced as to create an almost spatially continuous mosaic over a given region.

Figure 1 provides two examples of mosaics created with images from individual sequences.

During the $4^{\text {th }}$ periaxis passage (PJ4), JIRAM acquired 21 sequences of 13 images each over the north pole and 6 sequences of 19 images each over the south pole. The time interval between two consecutive sequences was 16 minutes over the north pole and 20 minutes over the south pole. The best pixel spatial resolution at the reference 1-bar level varies from 100 to $9.6 \mathrm{~km}$ because of the high eccentricity of Juno's orbit. Similarly, the mean emission angle within a given sequence varies from $75^{\circ}$ to $3^{\circ}$. In the analysis presented here we considered only sequences with a spatial resolution better than $40 \mathrm{~km}$. An exception was made for sequence 170202_144131, to get at least three sequences over the south pole. The emission angle also needs to be limited, to cope with uncertainties on the radiance emission angle correction in these unexplored cloud conditions. The limit was arbitrarily set to $40^{\circ}$.

Table 1 lists the sequences selected for this study.

Geometric parameters of each image (geographic locations of pixel corners and centers, solar zenith, emission and phase angles, slant distance, etc.) were computed by means of the SPICE Toolkit [Acton, 1996, Action et al., 2017] on the basis of the re-constructed kernel files available at https://naif.jpl.nasa.gov/pub/naif/JUNO/kernels/. The nominal pointing uncertainty of JIRAM images is about one pixel, translating into minimum pointing uncertainties of about $12 \mathrm{~km}$ for the data considered in this study.

\section{Methods}

The winds were estimated by tracking the motions of different atmospheric features (clouds) over frames acquired at different times. This method relies on the assumption that changes in feature 
positions are caused solely by the motion of air parcels instead of other phenomena such as condensation/sublimation (notably, due to the propagation of waves).

The wind motions were tracked by minimizing the mean absolute distortion (MAD), as defined in eq. 8.2-37 of Gonzalez \& Woods [2008]. The method compares two images taken at different times. Firstly, the images are re-interpolated over a common bi-dimensional sampling grid (in our case, with a uniform sampling step of $11.6 \mathrm{~km}$ in both spatial directions, corresponding to 0.01 degrees in latitude). Then, for a neighborhood of each point of the first image, the our algorithm finds the bidimensional displacement vector that better matches what is observed in the second image (in our case, the neighborhood of a given point is defined as the square $21 \times 21$ pixels image section centered over the point). This allows one, on the basis of the time elapsed between acquisitions, to compute a bi-dimensional speed vector for each point of the first image, creating therefore a 'speed field'. Notably, the retrieved speed fields have an effective spatial resolution comparable to the adopted size of search neighborhood, therefore values reported in this paper shall be interpreted as mean air speeds averaged over an area of about 240x240 km around the point of interest. From here on we will use the term 'amplitude' to indicate the length of a speed vector. Further details are provided in Appendix 1 in Supporting Information.

The estimate of displacements between images are performed in terms of integer numbers of sampling steps along both axes. This implies a minimum detectable motion of 1 sampling step (11.6 $\mathrm{km})$. For images acquired 16 minutes apart - such as the north pole pairs presented in section 4 this corresponds to a minimum detectable speed of $12 \mathrm{~m} / \mathrm{s}$; this value reduces to $9.6 \mathrm{~m} / \mathrm{s}$ for the south pole pairs acquired 20 minutes apart. These values represent the expected amplitude of random error on our wind estimates, that affects independently each point of the retrieved speed fields.

Conversely, systematic speed errors are those affecting by the same amount the entire velocity field computed from a given pair of images, adding a uniform (over space) unknown vector in the retrieved velocity field. They are mostly caused by the limited precision achievable in pointing reconstruction. Eventually, pointing uncertainties on re-interpolated images can produce apparent shifts of atmospheric features over the assumed geographical grid - and therefore additional spurious components in speed fields - even in the absence of any intrinsic air parcel motion. Exploiting the large degree of spatial overlap between individual images inside each of the sequences listed in table 1 , we were able to develop a method to compare the speeds retrieved from different pairs of images and to remove at least the relative differences in the systematic errors between different parts of the mosaics of speed maps. Details are provided in Appendix 2 in Supporting Information.

The six north-pole sequences were arranged in pairs, in order to achieve a time gap around 16 minutes between the selected components of a pair. Pairs with a shorter time gap would have a 
correspondingly greater minimum detectable wind speed $(24 \mathrm{~m} / \mathrm{s}$ for the 8 -minute case). Pairs with a longer time gap would require computational times for the algorithm exceeding our currently available capabilities. Moreover, longer time intervals also increase the possibility of occurrence for phenomena that cannot be accounted for by the MAD criterion, such as small-scale rotations and changes of the cloud patterns due to wave activity. For the three south pole sequences, the only options were pairs with a time gap around 20 minutes between the selected components.

Table 2 details the sequence pairs used for speed fields determination.

\section{Results}

\subsection{Preliminary analysis}

The retrieved velocity field for the N1 case - in reference to the System III coordinate system - is shown in Figure 2.

A first notable feature is the azimuthal variation of the velocity amplitude around the NPC centre (approximatively located at $\left[89.5^{\circ} \mathrm{N}, 130^{\circ} \mathrm{E}\right]$ ), that tends to diminish considerably along the $0^{\circ}$ longitude meridian and to increase along the $180^{\circ}$ longitude meridian. In each point within 2 degrees latitude from NPC centre, the direction of wind remains essentially orthogonal to the line joining the considered point with V1 centres, with no significant radial components, making it unlikely that local decreases/increases of wind speeds are related to a Rossby wave. On the other hand, visual inspection of NPC cloud features as imaged by JIRAM (Figure 3) demonstrates a remarkable degree of axial symmetry (in terms of typical signal amplitudes and frequency of variations at fixed distance from the centre) up to about $1700 \mathrm{~km}$ from NPC centre. Moreover, the wind direction in regions of low velocity amplitude around NPC (namely, those about the $87^{\circ}$ latitude circle) as seen in Figure $2 \mathrm{~b}$ appears to be systematically oriented toward the approximately $90^{\circ} \mathrm{W}$ meridian (negative $\mathrm{x}$ direction).

\subsection{Introduction of a symmetrization procedure}

These facts possibly can be explained assuming the existence of a systematic component in the retrieved speed field presented in Figure 2. This component would be a single bi-dimensional vector, uniform over the speed field or at least in large areas around NPC and approximatily oriented along the $\mathrm{x}$-axis. It should be stressed that this systematic component can be caused by a pointing error or by an actual motion of NPC with respect to the System III coordinate or by a combination of the two. Ideally, its removal from the retrieved field should be able to compensate at the same time systematic directions observed in weak wind areas as well as the azimuthal speed asymmetry around NPC centre. This latter feature is particularly desirable, since azimuthal symmetric speed fields can more easily justify the long-time persistence of NPC, that was again observed with similar size, position and appearance in JIRAM images from PJ5 and PJ6.

In practical terms, the removal of the systematic component consists in the subtraction of a uniform 
velocity vector (a pair of uniform components along $\mathrm{x}$ and $\mathrm{y}$ axes) from the entire velocity field returned by the procedure described in Appendix 2 in Supporting Information, without the introduction of any additional rotation or further transformation.

The criterion that we chose to adopt to compute the systematic component was the maximization of axial symmetry of velocity amplitudes around NPC, upon removal of the searched component from the original speed field. This was achieved by minimizing the total standard deviation of the speed amplitude, as computed over several annuli around the apparent NPC centre up to a distance of about $1500 \mathrm{~km}$. This method is formally equivalent to searching, in each annulus, for the systematic component that, upon subtraction, brings the velocity amplitudes as close as possible to their mean value. This symmetrization procedure - that introduces a (uniform over the field) correction of the speed field - has been exploited extensively in our subsequent analysis to determine the mean vortice's motions with respect to System III. Notably, the symmetrization procedures relies on a large number of independent wind speed estimates around the vortex center and therefore exploits correlations among independent measurements. Numerical tests have demonstrated that variations in the order of $2.5 \mathrm{~m} / \mathrm{s}$ on the systematic component to be removed are enough to introduce detectable variations in the derived total standard deviation of the speed amplitudes used to estimate the degree of azimuthal simmetry.

The removal of the component of systematic motion due to the actual motion of NPC with respect to System III is conceptually equivalent to putting oneself in a reference frame, in motion with respect to System III, where the vortex centre remains stationary and the speed field become axially symmetric around that centre.

In the case of field $\mathrm{N} 1$, corrections of $\mathrm{v}_{\mathrm{x}}=-14 \mathrm{~m} / \mathrm{s}$ and $\mathrm{v}_{\mathrm{y}}=0 \mathrm{~m} / \mathrm{s}$ were deemed adequate to make NPC axially symmetric (Figure 4a and A1). This correction is indeed capable of effectively nullifying velocity amplitudes around the $87^{\circ}$ latitude. For the other north pole cases N2-N4, the same symmetrization procedure adopted for NPC provided corrections on the x-component of the winds between -33 and $-28 \mathrm{~m} / \mathrm{s}$ and on the y-component of the winds between -1 and $5 \mathrm{~m} / \mathrm{s}$. These values are listed in the last two columns of Table 2. We reiterate that these corrections for the N1-N4 cases may include the compensations for a systematic genuine motion of NPC with respect to the System III coordinate system (that must be constant for the different cases, given the short time elapsed between different sequences) as well as for the pointing errors (variable from case to case). Albeit these two effects cannot in principle be disentangled and despite the extremely limited statistics (just four cases), we notice that along the $\mathrm{x}$ axis we have inferred consistently correction components above the uncertainty level toward the negative direction, suggesting therefore the occurrence of an actual motion of the NPC with respect to the System III coordinates along the $90^{\circ} \mathrm{W}$ direction. Figures 4 and A2 present the wind speeds measured over the north pole region in the four available fields, once the removal of systematic components to obtain symmetry for the 
central pole NPC has been applied to the entire corresponding field. Figure 5 and A3 present the same quantities for the south pole, after symmetrization for SPC.

\subsection{Revised analysis of NPC after symmetrization procedure}

At the north pole, NPC shows the highest wind speed amplitude of $75 \mathrm{~m} / \mathrm{s}$ at about 1,000 km from the vortex centre (Figure 6a). The mean relative vorticity - twice the azimuthal velocity divided by the radius - is therefore $1.5 \cdot 10^{-4} \mathrm{~s}^{-1}$, which is about half the planetary vorticity at the pole of $3.5 \cdot 10^{-4}$ $\mathrm{s}^{-1}$ and of the same sign. The NPC centre has an offset from the nominal Jupiter rotation pole (albeit not as evident as observed for the SPC), with offset estimates varying between 0.4 (from photometric estimate of signal symmetry of mosaic 170202_120653) and 0.15 (from location of wind speed minima in N3) latitude degrees. Despite the adopted symmetrization procedure, the wind field retains some asymmetry around speed minima. Namely, the speed peak appears consistently larger in width and slightly lower in peak value along the positive $\mathrm{x}$ and $\mathrm{y}$ directions (i.e.: along the $135^{\circ} \mathrm{E}$ meridian). This asymmetry is possibly related to the presence of the small "A" vortex along the $120^{\circ} \mathrm{E}$ meridian. Wind speed sections from different fields are essentially consistent once the nominal error of $12 \mathrm{~m} / \mathrm{s}$ is kept in mind.

\subsection{Possible evidence of relative motions between cyclones in the north polar region}

Among the four internal vortices around NPC, only two (CPCn6 and CPCn2) are covered by our maps. We should note that symmetrization of the wind field for NPC did not lead to axial symmetric velocity fields for these two features, which appear weaker on the east sides for both vortices. Moreover, the velocity patterns in Figures 4 and A2 around $\mathrm{CPCn} 6$ and $\mathrm{CPCn} 2$ are quite constant from $\mathrm{N} 1$ to $\mathrm{N} 4$, suggesting that axial asymmetry of CPCn6 and CPCn2 is not an observational artfact. The axially symmetric appearance of both features in JIRAM images as well as their stability over several Juno orbits points again toward the occurrence of relative motions of the two vortices with respect to NPC centre, such that the velocity fields appear indeed axially symmetric in the reference frames moving along their own centres. Moving from this assumption, we repeated the symmetrization procedure described above separately for both CPCn6 and CPCn2 vortices, retrieving the corresponding corrections. Once this correction is subtracted from the correction provided by the symmetrization of NPC, we can infer the net relative velocity of each feature with respect to NPC. This study is best performed in the N1 case, because of the continuous spatial coverage offered by this speed field. Results are reported in Table 3. Since speeds for both CPCn6 and CPCn2 refer to NPC, difference between these components provides also the relative motion of the two circumpolar cyclones.

Unfortunately, it is not possible to verify independently the relative motions of cyclones by tracking their centres over JIRAM images acquired at different times. The large uncertainties on the definition of vortex 'centres', regardless of the adopted method (radiometric barycentres or minima 
of speed field), implies errors on derived feature speeds much larger than those achieved by speed axial symmetrization. For example, in the NPC case the position of centre can be defined with an uncertainty of 0.15 degrees in longitude: this implies an uncertainty on speeds of $180 \mathrm{~m} / \mathrm{s}$ for the northern cases listed in Table 3, about an order of magnitude greater than the values reported in Table 3.

The magnitude of speed of CPCn6 presented in Table 3 around NPC would imply a rotation period of about $310^{6} \mathrm{~s}$, i.e: about 85 Jupiter rotation periods or 35 Earth days. However, net speeds of CPCn6 and CPCn2 with respect to NPC are neither oriented along the parallels (i.e.: both have a meridional component) nor consistent with a rigid rotation of the entire pattern of nine northern vortices around the pole, since both cyclones appears to move approximatively toward the $150 \mathrm{~W}$ meridian. JIRAM images of the north polar region acquired during subsequent JIRAM pericenter passages (from PJ5 to PJ10, in December 2017) clearly show the same pattern of cyclones presented in Fig. 1a, with circumpolar cyclones with distinctive morphologies (notably CPCn4) at approximatively the same positions. This fact strongly supporting the long-time stability (in the time scale of tents of months) of these structures as well as the fact that relative motions presented in Table 3 are either irregular in time or subject to periodic reversals. Unfortunately, during later pericenter passages overlap of JIRAM image sequences over the north pole was not adequate to retrieve independent speed fileds such those presented in Fig. 4.

\subsection{CPCs and othe features in the north polar region}

The sizes of CPCn6 and CPCn2, as inferred from wind speed fields, are comparable to that of NPC, but absolute peak speeds (even after symmetrization) are considerably lower, reaching about $55 \mathrm{~m} / \mathrm{s}$ again at about $1000 \mathrm{~km}$ from their apparent centers.

The small "A" feature is an anticyclonic feature and presents maximum speeds around $42 \mathrm{~m} / \mathrm{s}$. At least four further smaller anticyclonic structures exist around NPC (two are seen Figure 7a and a further one in Figure 7b, marked as "a" ).

Results on CPCn3 and CPCn7 are more problematic. Firstly, their marginal positions on N1 did not allow a symmetrization to be performed to compensate the joint effects of the motion of the feature with respect to System-III coordinates and of pointing errors. Secondly, significant portions of external rims (beyond $2^{\circ}$ from their respective apparent centres, regions hosting both very dark and very bright areas in Figure 1a) are essentially featureless, strongly limiting the validity of motion tracking by mean of MAD estimate. Despite these limitations, some results are robust: their central parts (at intermediate brightness in Figure 1a) are rich in features, that allow effective tracking of wind speeds. The fastest annulus on CPCn3 at about $1000 \mathrm{~km}$ from cyclone center shows speeds in the range $100-110 \mathrm{~m} / \mathrm{s}$. The mean relative vorticity is therefore around $2 \cdot 10^{-4} \mathrm{~s}^{-1}$, to be compared against a planetary vorticity at $82.5^{\circ}$ of $3.47 \cdot 10^{-4} \mathrm{~s}^{-1}$ and of the same sign. Coverage of CPCn7 is less complete and the area corresponding to the high-speed annulus in CPCn3 is not included in N1. 
Nevertheless, the region where estimates are available is already suggestive of much higher speeds than the ones observed in NPC, CPCn6 and CPCn2. An interesting feature associated with CPCn3 is located at $\left[85^{\circ} \mathrm{N}, 90^{\circ} \mathrm{E}\right]$ where velocity maps, supported by visual inspection, demonstrate how the flow is strongly accelerated (Figure $7 \mathrm{~b}$, marked as " $\mathrm{f}$ "), moving from the stagnant regions at $\left[83^{\circ} \mathrm{N}, 105^{\circ} \mathrm{E}\right]$.

\subsection{Cyclones in the south polar region}

Over the south pole, symmetrization of velocity fields around SPC provided - for both the S1 and S2 cases - correction values that once extended to the entire corresponding speed field provided rather symmetric values also around the centres of CPCs3, CPCs5 and CPCs1. The symmetrization procedure for the two cases $\mathrm{S} 1$ and $\mathrm{S} 2$ returned similar corrections, possibly suggestive of an overall motion of most of the south pole six vortices pattern toward the approximate direction of $60^{\circ} \mathrm{E}$. The amplitudes of the corrections are, however, rather small once compared to the expected systematic uncertainties on speed determination due to the pointing, and consequently the values reported in Table 2 should actually be interpreted as upper limits for the overall motions of south pole vortices. Notably, the computation of total standard deviation of the velocity amplitudes implied by the symmetrization procedure - was limited to distances from nominal SPC center entirely covered by available velocity maps, to avoid biases related to vicinity of image borders.

It should be noted that even a simple visual inspection of Figure $1 \mathrm{~b}$ demonstrates the asymmetric nature of SPC: its central part appears clearly elongated along the $150^{\circ} \mathrm{E}$ meridian and its centre shows an offset of about $1.5^{\circ}$ from the nominal south pole. It is therefore not surprising to observe a residual asymmetry of speed amplitudes even after the symmetrization of speed amplitudes had been performed at the best degree allowed by the removal of a uniform vector in the entire SPC region. With comparison to NPC, the speed sections (Figure 6b) present higher peaks values (up to $90 \mathrm{~m} / \mathrm{s}$ at $1,000 \mathrm{~km}$ from the centre), and a slightly higher relative vorticity of $1.8 \cdot 10^{-4} \mathrm{~s}^{-1}$.

CPCs4, even after an attempt to symmetrize separately speed amplitudes around its apparent centre, shows a very peculiar structure, with two arms at moderate speed (approximately $55 \mathrm{~m} / \mathrm{s}$, indicated by " $\mathrm{m}$ " in Figure 5a) apparently embracing a relatively stagnant area at [ $\left.84^{\circ} \mathrm{S}, 25^{\circ} \mathrm{W}\right]$ ("s" in Figure 5a). Larger images of the same area strongly suggest that the upper arm is associated with an apparent acceleration of the flow from an area located at $\left[80^{\circ} \mathrm{S}, 45^{\circ} \mathrm{W}\right]$ (just west of area "J" in Figure 4 of Adriani et al. [2017]).

CPCs5 presents the highest wind speeds observed in Jupiter polar vortices, exceeding $115 \mathrm{~m} / \mathrm{s}$. No evident high speed arms were detected for this vortex, as well as for CPCs1, despite their morphological resemblance (very opaque centre) with CPCs4. CPCs3 presents some small scattered areas of apparent low speed along its high-speed annulus (seen as blue specks within CPCs5 in Figure 5a), but these turned out to be associated with featureless regions in Figure $1 \mathrm{~b}$ and are therefore likely related to algorithm failures. 


\subsection{Other features in the south polar region}

The south polar region presents a number of other interesting features. An irregular ribbon of moderate wind $(55 \mathrm{~m} / \mathrm{s})$ exists around $\left[83^{\circ} \mathrm{S}, 15^{\circ} \mathrm{E}\right]$ (rightmost " $\mathrm{r}$ " label in Figure 5a), surrounding a very opaque area centred at $\left[81^{\circ} \mathrm{S}, 15^{\circ} \mathrm{E}\right]$. In the entire region, winds appear to rotate clockwise, as observed for the main southern vortices (CPCs) described above. Figure 7c provides further details of the area (a part of the ribbon is marked there as " $r$ "). A second similar ribbon exists between $30^{\circ} \mathrm{E}$ and $45^{\circ} \mathrm{E}$ (leftmost " $\mathrm{r}$ " label in Figure 5a). Both ribbons represents likely the southern parts of highly distorted cyclonic structures, only partially covered in our velocity maps, possibly smaller versions of the folded filamentary regions reported in Orton et al, 2017 at lower latitudes in JunoCam data

A number of small anticyclones, with very opaque centres, are observed at $\left[85^{\circ} \mathrm{S}, 130^{\circ} \mathrm{W}\right],\left[82^{\circ} \mathrm{S}\right.$, $40^{\circ} \mathrm{W}$ ] (also visible in Figure $7 \mathrm{c}$ and marked there as "a") and $\left[81^{\circ} \mathrm{S}, 175^{\circ} \mathrm{W}\right]$. The small bright feature with a more opaque centre at $\left[82.5^{\circ} \mathrm{S}, 125^{\circ} \mathrm{W}\right]$ also appears to be an anticyclone. Extremely weak anticyclonic circulation is tentatively identified in two regions with low wind speeds $(<20$ $\mathrm{m} / \mathrm{s}$ ) between SPC, CPCs5 and CPCs4 (Figure 7d, marked as “a?"), but with patterns much less defined in comparison to what is observed around NPC.

\subsection{Velocity vs. radius beyond the vortex centre}

The NPC has a constant angular speed at least out to $500 \mathrm{~km}$ from vortex centre. Beyond $1400 \mathrm{~km}$, the decay of the tangential speed may be modelled as

$v=C_{1} \cdot r^{-\gamma}[1]$

Here $v$ is the tangential velocity, $r$ is distance from the vortex centre, and $\mathrm{C}_{1}$ is a constant. We observe (Figure 8a) that speeds decay more quickly than expected for the case of an irrotational vortex, for which $\gamma=1$. A fit of speed data between 1500 and $3200 \mathrm{~km}$ returns a value of $\gamma=1.40 \pm 0.04$. The SPC from $2000 \mathrm{~km}$ to $2600 \mathrm{~km}$ displays a trend of speed with $\gamma=1.06 \pm 0.18$. Beyond a radius of $2,600 \mathrm{~km}$, the tangential speed drops more quickly with increasing distance, with $\gamma$ approaching the value of 1.5 (Figure $8 \mathrm{~b}$ ). These profiles differ from a Rankine-type vortex because the latter has $\gamma=1$ (vorticity $=0$ ) outside a circular region of constant vorticity.

\section{Discussion}

With the NPC centre as a reference, symmetrization of the wind field in the region within $3000 \mathrm{~km}$ yields a vortex that is essentially circular, i.e., the detected radial motions are comparable to the estimated speed error. For a circular vortex that is small compared to the planet's radius, cyclostrophic balance applies and the radial force balance in pressure coordinates is

$-\frac{v^{2}}{r}-f v=-g\left(\frac{\partial z}{\partial r}\right)_{P}[2]$ 
Here $f$ is $2 \Omega \sin \boldsymbol{\phi}, \boldsymbol{\phi}$ is latitude, $\Omega$ is the planet's angular velocity, and $z(r, P)$ is the height of a constant pressure surface. For the NPC the ratio of the first and second terms is 0.21 , meaning that the Coriolis acceleration dominates and the force balance is nearly geostrophic.

Assuming $v$ is a linear function of $r$ from zero at the centre to $75 \mathrm{~m} \mathrm{~s}^{-1}$ at $\mathrm{r}=1000 \mathrm{~km}$, the height $z$ at $r=1000 \mathrm{~km}$ compared to that at the centre is $640 \mathrm{~m}$. This is about 0.025 times the pressure scale height at the 1-bar level, where the temperature is $165 \mathrm{~K}$. The mean relative vorticity $\zeta$, which is the line integral of velocity around the circumference divided by the area, is $2 v / r$, and the vortex Rossby number $\zeta / f[$ Legarreta and Sanchez-Lavega, 2005] is Ro $=2 \mathrm{v} /(\mathrm{rf})$. Thus Ro $=0.43$ for the NPC.

Other vortices on Jupiter [Vasavada et al., 1998, Choi et al., 2007, Simon-Miller et al. 2002, Hueso et al. 2009, Sussman et al. 2010] have wind speeds that are comparable to the $\sim 100 \mathrm{~m} \mathrm{~s}^{-1}$ speeds of the polar vortices. Legarreta and Sanchez-Lavega [2005] performed an extensive study to measure the vorticity of Jupiter cyclones and anticyclones. They report absolute values of Ro in the range from $\sim 0.2$ to 0.5 . For the Great Red Spot and White Ovals the values are 0.30 and 0.33 , respectively. The NPC value is consistent with the other vortices, although it is on the high side of the distribution. The vorticity itself, which is $15 \cdot 10^{-5} \mathrm{~s}^{-1}$, is higher than all but one of the values reported by Legarreta and Sanchez-Lavega, but it is still well below the planetary vorticity of $35 \cdot 10^{-}$ ${ }^{5} \mathrm{~s}^{-1}$.

Tropical cyclones on Earth have wind speeds similar to those of the NPC and SPC, but the radius of maximum wind is much smaller. A category 5 hurricane is defined as $v \geq 70 \mathrm{~m} \mathrm{~s}^{-1}$. A typical radius for the "eye," where the winds are strongest, is $25 \mathrm{~km}$. At $\boldsymbol{\phi}=30^{\circ}$, corresponding to the south coast of the USA, the Rossby number is 39, meaning that the Coriolis acceleration in equation [2] is almost negligible. Despite this huge difference between cyclones on Earth and Jupiter, the height variation of a constant pressure surface relative to the scale height is about the same, 0.030 and 0.025 respectively.

The polar cyclones on Saturn have about the same size and speed as those on Jupiter (Dyudina et al., 2009), but there is only one cyclone at each pole. This is a significant difference between the two planets that has yet to be explained. The radius of the eye is $\sim 1000 \mathrm{~km}$, the atmosphere within the eye is warmer than that outside, and the eye wall clouds are 1-2 scale heights above the clouds in the centre.

Adriani et al. [2017] observe that the constellation of CPCs is reminiscent of vortex crystals, which occur in strictly two-dimensional (2D) flows. Starting with Lord Kelvin [Thomson, 1878], many authors have studied laboratory-scale mechanical systems that exactly simulate the 2D motion of columnar vortices [Mayer, 1878; Campbell and Ziff, 1979; Fine et al., 1995]. The laboratory results have been verified by numerical integration of the 2D Euler equations, with a variety of forcings, boundary conditions, and vorticity distributions [Schecter et al., 1999; Jimenez and Guegan, 2007]. The common feature is the 2D nature of the flow, where the velocity around an isolated vortex falls off as $1 / \mathrm{r}$, the streamfunction grows as $\log (\mathrm{r})$, and the circulation is a constant out to infinity. 
These are long-range interactions. Planetary atmospheres are more complicated. Moderate vertical stratification or simply a free surface that moves up and down produce a finite radius of deformation, which limits the range of interaction. Rotation and spherical geometry produce a background vorticity gradient, a beta effect, which allows planetary wave propagation [Vallis, 2007]. These effects are small close to the pole if the stratification is strong enough, and that could help explain the existence of Jupiter's polar vortices.

The $1 / \mathrm{r}^{\gamma}$ falloff with distance shown in Fig. 8, where $\gamma$ is $1.4-1.5$, is not inconsistent with a strictly 2D flow. It simply means that that NPC and SPC are surrounded by fluid with anticyclonic vorticity. The vorticity associated with a $1 / \mathrm{r}^{\gamma}$ falloff is proportional to $(1-\gamma) / \mathrm{r}^{\gamma+1}$, which is negative when $\gamma>$ 1. If one uses a straight line fit near the outer region, one would conclude that the velocity is zero at $\mathrm{r}=4000 \mathrm{~km}$ for the NPC and is zero at 4500 for the SPC. Then the circulation would be zero at those distances, and one would have a "shielded" vortex, in which the spatially integrated vorticity is zero. Such behaviour is inconsistent with a laboratory model [Fine et al., 1995; Schecter et al., 1999] that has only one sign of vorticity, but it is consistent with a $2 \mathrm{D}$ simulation [Jimenez and Guegan, 2013] that has both signs of vorticity and a spatial average of zero.

\section{Conclusions}

Results presented here are a first attempt to extract wind fields over large portions of Jupiter polar areas, with random errors in the order of $12 \mathrm{~m} / \mathrm{s}$. Data were acquired during a single Juno pericenter passage on February $2^{\text {nd }}, 2017$.

The two cyclones placed over both poles (NPC and SPC) reach their maximum speeds at about $1,000 \mathrm{~km}$ from their respective centers; the southern vortex appears to rotate slightly faster that the northern counterpart $(90 \mathrm{~m} / \mathrm{s}$ and $75 \mathrm{~m} / \mathrm{s}$ respectively). In the surrounding circumpolar cyclones, speed exceeds $100 \mathrm{~m} / \mathrm{s}$ in at least three cases (CPCn3, CPCs1, CPCs5). Considering the other cicrcumpolar cyclones included in our maps, the two southern structures (CPCs4 and CPCs3) show sligthly lower maximum speeds $(80-90 \mathrm{~m} / \mathrm{s})$ while the two northern vortices (CPCn2 and CPCn6) appear considerably slower (about $55 \mathrm{~m} / \mathrm{s}$ ). In all cases, the peak speeds are reached at about 1000 $\mathrm{km}$ from their respective centers, but in general peaks are rather broad, and therefore the definition of the size of maximum speed ring is prone to an uncertainity of about $500 \mathrm{~km}$.

Central polar vortices in both hemispheres are in moderate cyclostrophic balance and with velocities increasing linearly along radius within $500 \mathrm{~km}$ from their centres. In the outer parts of the southern vortex, flow is irrotional in a large annulus $(\gamma=1.06 \pm 0.18)$; in the northern vortex, rotational speed decays along radius faster than expected for irrotational flow $(\gamma=1.40 \pm 0.04)$.

While the main vortices are confirmed to be cyclones, several smaller anticyclonic features are also detected.

We made azimuthal averages around vortex centres, to account both for pointing errors as well as 
systematic motions of vortices with respect to the System-III coordinates. Despite the ambiguity related to the absolute pointing, both polar cyclones appear to be subject to overall motions with respect to System III, with translation speeds of the vortex centres in the order of $25 \mathrm{~m} / \mathrm{s}$ for the northern cyclone and slightly less $(18 \mathrm{~m} / \mathrm{s})$ for the southern cyclone. The stability of vortex patterns over several Juno perijove passages implies, however, that these motions are not constant and may reverse between Juno perijoves.

\section{Acknowledgments}

Original JIRAM data used for this work are available at the NASA Planetary Data System web site https://pds-atmospheres.nmsu.edu/data_and_services/atmospheres_data/JUNO/jiram.html

This work was supported by the Italian Space Agency through ASI-INAF contract I/010/10/0 and 2014-050-R.0.

JIL and SKA acknowledge support from NASA through the Juno Project.

API was supported partly by the Juno Project of NASA and partly by the US National Science Foundation (NSF Grant 1411952).

GSO acknowledges support from NASA through funds that were distributed to the Jet Propulsion Laboratory, California Institute of Technology.

The JIRAM instrument has been developed by Leonardo at the Officine Galileo - Campi Bisenzio site.

The JIRAM instrument was conceived and brought to reality by our late collaborator and institute director Dr. Angioletta Coradini (1946-2011).

\section{References}

Acton, C. H. (1996), Ancillary Data Services of NASA's Navigation and Ancillary Information Facility, Planetary and Space Science, 44 (1), pp. 65-70.

Acton, C.H, et al. (2017), A look toward the future in the handling of space science mission geometry, Planetary and Space Science, DOI 10.1016/j.pss.2017.02.013

Adriani, A. et al. (2014), JIRAM, the Jovian Infrared Auroral Mapper Space Sci Rev, doi:10.1007/s11214-014-0094-y

Adriani, A. et al. (2018), Geometric cyclonic patterns in Jovian Polar Regions, Nature, 555, doi:10.1038/nature25491 
Asay-Davis, X. S., et al. (2009), Jupiter's shrinking Great Red Spot and steady Oval BA: Velocity measurements with the 'Advection Corrected Correlation Image Velocimetry' automated cloudtracking method, Icarus, 203, 164-188, doi: 10.1016/j.icarus.2009.05.001

Atreya, S. K., et al. (1999), Comparison of the atmospheres of Jupiter and Saturn: deep atmospheric composition, cloud structure, vertical mixing, and origin. Planet. Space Sci., 47, 124362. doi:10.1016/S0032-0633(99)00047-1

Baker, A. L., et al. (1975) The Imaging Photopolarimeter Experiment on Pioneer 11, Science, 188 (4187), 468-472, doi: 10.1126/science.188.4187.468

Barrado-Izagirre, N., et al. (2008), Jupiter's polar clouds and waves from Cassini and HST images: 1993-2006, Icarus, 194, 173-185, doi: 10.1016/j.icarus.2007.08.025

Bolton, S. J., et al. (2017), Jupiter's interior and deep atmosphere: The initial pole-to-pole passes with the Juno spacecraft. Science, 356 (6340), 821-825, doi: 10.1126/science.aal2108

Campbell, L. J., and Robert M. Ziff (1979) Vortex patterns and energies in a rotating superfluid Physical Review B, 20, 1886-, doi: 10.1103/PhysRevB.20.1886

Choi, D. S., et al. (2007), Velocity and vorticity measurements of Jupiter's Great Red Spot using automated cloud feature tracking, Icarus, 188, 35-46, doi: 10.1016/j.icarus.2006.10.037

Fine, K. S., et al. (1995) Relaxation of 2D turbulence to vortex crystals, Physical review letters, 75 , 3277-, doi: 10.1103/PhysRevLett.75.3277

Garcìa-Melendo, E., Sánchez-Lavega, A. (2001), A Study of the Stability of Jovian Zonal Winds from HST Images: 1995-2000, Icarus, 152, 316-330, doi: 10.1006/icar.2001.6646

Geng, Q., and Sugi, M. (2003), Possible Change of Extratropical Cyclone Activity due to Enhanced Greenhouse Gases and Sulfate Aerosols-Study with a High-Resolution AGCM, Journal of Climate, 13 (16), 2262-2274, doi: 10.1175/1520-0442(2003)16<2262:PCOECA>2.0.CO;2

Gonzalez, R. C., Woods, R. E. (2008), Digital image processing, $3^{\text {rd }}$ edition. Prentice Hall, ISBN 13: 978-0-13-505267-9

Grassi, D., et al. (2017a), Preliminar yresults on the composition of Jupiter's troposphere in hot spot regions from the JIRAM/Juno instrument, Geophys. Res. Lett., 44, doi:10.1002/2017GL072841

Grassi, D., et al. (2017b), Analysis of IR-bright regions of Jupiter in JIRAM-Juno data: Methods and validation of algorithms, Journal of Quantitative Spectroscopy and Radiative Transfer, 202, doi:10.1016/j.jqsrt.2017.08.008

Hueso, R., and A. Sánchez-Lavega (1998), Motions in hot spot-plume regions using Voyager images, Icarus, 136, 353-357, doi:10.1006/icar.1998.6018 
Hueso, R., et al. (2009), The jovian anticyclone BA: II. Circulation and interaction with the zonal jets, Icarus, 203 (2), 499-515, doi: 10.1016/j.icarus.2009.05.004

Hueso, R., et al. (2017), Jupiter cloud morphology and zonal winds from ground observations before and during Juno's first perijove, Geophys. Res. Lett., 44, 4669-4678, doi:10.1002/2017GL073444

Ingersoll, A. P., et al. (1981), Interaction of eddies and mean zonal flow on Jupiter as inferred from Voyager 1 and 2 images, J. Geophys. Res., 86(A10), 8733-8743, doi:10.1029/JA086iA10p08733

Irwin, P. G. J., et al. (2001), The Origin of Belt/Zone Contrasts in the Atmosphere of Jupiter and Their Correlation with 5- m Opacity, Icarus, 149, 397-415, doi:10.1006/icar.2000.6542.

Jimenez, J. and A. Guegan (2007), Spontaneous generation of vortex crystals from forced twodimensional homogeneous turbulence, Phys. Fluids 19, 085103; doi: 10.1063/1.2757713

Legarreta, J., Sánchez-Lavega, A. (2005), Jupiter's cyclones and anticyclones vorticity from Voyager and Galileo images, Icarus, 174 (1),178-191, doi: 10.1016/j.icarus.2004.10.006

Lee, D.T., Schachter, B.J. (1980), Two Algorithms for Constructing a Delaunay Triangulation, Int. J. of Computer and Information Sci., 9, pp. 219-242

Li, R. et al. (1994), A new three-step search algorithm for block motion estimation, IEEE Transactions on Circuits and Systems for Video Technology, 4 (4), 438-442, doi: $10.1109 / 76.313138$

Limaye, S. S (1986), Jupiter: New estimates of the mean zonal flow at the cloud level, Icarus, 65, 335-352, doi: 10.1016/0019-1035(86)90142-9

Mayer, A. M. (1878), Floating magnets, Nature, 17 (442), 487-488

Orton, G. S., et al. (1981), Images of Jupiter from the pioneer 10 and pioneer 11 infrared

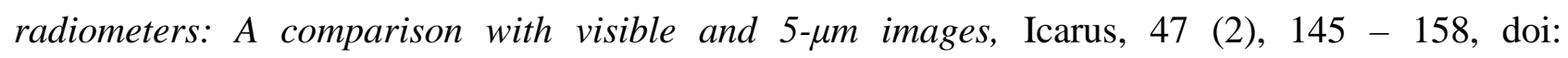
doi.org/10.1016/0019-1035(81)90162-7

Orton, G. S., et al. (2017), The first close-up images of Jupiter's polar regions: Results from the Juno mission JunoCam instrument, Geophys. Res. Lett., 44, 4599-4606, doi:10.1002/2016GL072443

Porco, C., et al., (2003), Cassini imaging of Jupiter's atmosphere, satellites, and rings. Science, 299 (5612), 1541-1547, doi: 10.1126/science. 1079462

Read, P. L., Gierasch, P. J., \& Conrath, B. J. (2006), Mapping potential Jupiter. II: the Great Red Spot from Voyager 1 and 2 data, Quarterly Journal of the Royal Meteorological Society, 132(618), 1605-1625, doi: 10.1256/qj.05.35 
Sánchez-Lavega, A., et al. (1998) A system of circumpolar waves in Jupiter's stratosphere, Geophys. Res. Lett., 25, 4043-4046, doi: 10.1029/1998GL900059

Schecter, D. A., et al. (1999) Vortex crystals from 2D Euler flow: Experiment and simulation Physics of Fluids, 11, 905-914., doi: 10.1063/1.869961

Simon-Miller, A. A., et al. (2002) New Observational Results Concerning Jupiter's Great Red Spot, Icarus, 158 (1), 249-266, doi: 10.1006/icar.2002.6867

Sussman, M. G., et al. (2010) Analysis of Jupiter's Oval BA: A streamlined approach, Icarus, 210 (1), 202-210, doi: 10.1016/j.icarus.2010.06.044

Thomson, W. (1878), Floating magnets, Nature 18 (444), 13-14

Vallis, G. K. (2017), Atmospheric and Oceanic Fluid Dynamics: Fundamentals and Large-Scale Circulation, Second Edition, Cambridge University Press, Cambridge, UK, doi: 10.1017/9781107588417, ISBN 978-1-107-06550-5

Vasavada, A. R., et al. (1998), Galileo Imaging of Jupiter's Atmosphere: The Great Red Spot, Equatorial Region, and White Ovals, Icarus, 135 (1), 265-275, doi: 10.1006/icar.1998.5984

Willert, C. E. and Gharib, M. (1991), Digital particle image velocimetry, Experiments in Fluids, 10 (4), 181-193, doi: 10.1007/BF00190388 


\section{Tables}

\begin{tabular}{|l|l|l|l|l|l|l|}
\hline Name & Pole & $\begin{array}{c}\text { Pixel resolution } \\
\text { range }(\mathrm{km})\end{array}$ & $\begin{array}{l}\text { Emission angle } \\
\text { range (degrees) }\end{array}$ & $\begin{array}{l}\text { Number } \\
\text { of images }\end{array}$ & $\begin{array}{l}\text { Latitude range } \\
\text { (degrees) }\end{array}$ & $\begin{array}{l}\text { Time coverage } \\
\text { (hhmmss UTC) }\end{array}$ \\
\hline $170202 \_114231$ & $\mathrm{~N}$ & $28.1-27.7$ & $17.13-37.14$ & 12 & $83.15 \mathrm{~N}-79.89 \mathrm{~N}$ & $114006-114541$ \\
\hline $170202 \_115039$ & $\mathrm{~N}$ & $24.6-23.7$ & $12.10-31.38$ & 12 & $83.49 \mathrm{~N}-80.46 \mathrm{~N}$ & $114813-115348$ \\
\hline $170202 \_115846$ & $\mathrm{~N}$ & $21.2-19.7$ & $5.36-24.50$ & 12 & $83.51 \mathrm{~N}-80.54 \mathrm{~N}$ & $115620-120155$ \\
\hline $170202 \_120653$ & $\mathrm{~N}$ & $17.9-15.8$ & $3.22-15.67$ & 12 & $82.85 \mathrm{~N}-79.99 \mathrm{~N}$ & $120426-121002$ \\
\hline $170202 \_121429$ & $\mathrm{~N}$ & $15.6-12.4$ & $2.83-25.93$ & 12 & $80.26 \mathrm{~N}-78.91 \mathrm{~N}$ & $121202-121738$ \\
\hline $170202 \_122236$ & $\mathrm{~N}$ & $17.7-9.6$ & $20.80-68.95 *$ & 12 & $64.11 \mathrm{~N}-78.52 \mathrm{~N}$ & $122007-122544$ \\
\hline $170202 \_140125$ & $\mathrm{~S}$ & $21.6-25.8$ & $3.57-19.10$ & 19 & $80.54 \mathrm{~S}-76.56 \mathrm{~S}$ & $135859-140808$ \\
\hline $170202 \_142113$ & $\mathrm{~S}$ & $30.5-33.2$ & $4.47-24.08$ & 19 & $79.37 \mathrm{~S}-79.89 \mathrm{~S}$ & $141846-142755$ \\
\hline $170202 \_144131$ & $\mathrm{~S}$ & $39.8-41.22$ & $10.46-35.15$ & 19 & $76.86 \mathrm{~S}-80.07 \mathrm{~S}$ & $143904-144813$ \\
\hline
\end{tabular}

Table 1: summary of JIRAM M-filter image sequences considered for this study. For each sequence, we list the range of mean pixel resolution and mean emission angle (as computed within individual images) found in the sequence. The sequence file name encodes the mean acquisition time of images according the scheme: yymmdd_hhmmss. Latitude range provides the latitude centers of images at the extremes of each sequence. Sequences of images pass always over the corresponding pole. Time coverage provides the acquisition times of images at the extremes of each sequence.

*Some images exceeding the $40^{\circ}$ mean-emission angle limit were excluded from analysis.

\begin{tabular}{|l|l|l|l|l|}
\hline Field name & First component & $\begin{array}{l}\text { Second } \\
\text { component }\end{array}$ & $\begin{array}{l}\text { Correction on } \begin{array}{l}\mathbf{x} \\
\text { component } \\
\text { speed }(\mathbf{m} / \mathbf{s})\end{array} \\
\text { of }\end{array}$ & $\begin{array}{l}\text { Correction on y } \\
\text { component } \\
\text { speed (m/s) }\end{array}$ \\
\hline N1 & $170202 \_114231$ & $170202 \_115846$ & -14 & 0 \\
\hline N2 & $170202 \_115039$ & $170202 \_120653$ & -33 & -1 \\
\hline N3 & $170202 \_115846$ & $170202 \_121429$ & -28 & 4 \\
\hline N4 & $170202 \_120653$ & $170202 \_122236$ & -30 & 1 \\
\hline S1 & $170202 \_140125$ & $170202 \_142113$ & 18 & 4 \\
\hline S2 & $170202 \_142113$ & $170202 \_144131$ & 18 & 15 \\
\hline Tablent
\end{tabular}

Table 2. Details of velocity fields presented in this study. The source JIRAM sequences are listed for the first and second component used for motion tracking. The last two columns provides the 
systematic corrections on $\mathrm{x}$ and $\mathrm{y}$ components of speed deemed necessary to make the central vortex axially symmetric (see section 4 for details).

\begin{tabular}{|l|l|l|l|l|}
\hline \multicolumn{2}{|l|}{} & \multicolumn{2}{|l|}{ CPCn6 } & \multicolumn{2}{l|}{ CPCn2 } \\
\cline { 2 - 5 } & Net $\mathbf{v}_{\mathbf{x}}(\mathbf{m} / \mathbf{s})$ & Net $\mathbf{v}_{\mathbf{y}}(\mathbf{m} / \mathbf{s})$ & Net $\mathbf{v}_{\mathbf{x}}(\mathbf{m} / \mathbf{s})$ & Net $\mathbf{v}_{\mathbf{y}}(\mathbf{m} / \mathbf{s})$ \\
\hline Field N1 & 16. & -3. & 2 & -7.5 \\
\hline
\end{tabular}

Table 3. Motion of CPCn6 and CPCn2 centers with respect to NPC, as derived imposing corrective speed components on wind fields to achieve approximate axial symmetry of wind fields (separately) around each vortex centre. 


\section{Figure captions}

Figure 1. Examples of mosaics composed from spatially interpolated images. Each mosaic contains only images from the JIRAM sequence given in the label. Further details on geometric properties of the observations are provided in Table 1. Labels identify individual features mentioned in the text according the nomenclature adopted in Adriani et al., 2018.

Figure 2: Wind velocity amplitudes (panel a) and directions (panel b) with respect to System-III coordinates, as derived in the N1 case . Background image for the left pane is a mosaic of the original images from the 170202_114231 sequence. Directions are presented only in the areas were overlap with images of sequence 170202_115846 exists.

Figure 3. Detailed view of the NPC region from sequence 170202_120653. Data were reprojected as follows: the distance of pixel along the y axis (radius) corresponds to its radial distance from the estimated vortex centre at $\left[89.6^{\circ} \mathrm{N}, 130^{\circ} \mathrm{E}\right]$, the distance of pixel along the $\mathrm{x}$ axis (theta) corresponds to the angle between the directions pixel-vortex centre and pole-vortex centre. This representation allows one to appreciate the degree of axial symmetry of vortex cloud structure up to at least 1.5 degree from vortex centre $(1750 \mathrm{~km})$.

Figure 4: Wind velocityamplitudes (panel a) and directions (panel b) over the north pole, derived for the N1 case of Table 2, as corrected to achieve maximum azimuthal symmetry of wind speed around the centre of NPC. Figure A2 in Supplemental Information provides analogous information for cases $\mathrm{N} 2, \mathrm{~N} 3$ and $\mathrm{N} 4$.

Figure 5: Wind velocity amplitudes (panel a) and directions (panel b) over the south pole, derived for the S2 case of Table 2, as corrected to achieve maximum azimuthal symmetry of wind speed around the centre of SPC. Labels " $\mathrm{m}$ ", "s" and " $\mathrm{r}$ " indicate specific features discussed in the text. Figure A3 in Supplemental Information provides analogous information for case S1.

Figure 6. Cross sections of wind velocity amplitudes fields along the $\mathrm{x}$ and $\mathrm{y}$ directions of Figure 1 for the central vortices over the north (panel a) and south (panel b) poles. Different colours are for different cases listed in table 2 and different directions.

Figure 7. Details from speed fields N1 (panels a and b) and S2 (panels c and d). "A" and "a" letters indicate anticyclonic areas. "a?" indicate possible locations of further anticyclonic areas. "f" and " $r$ " indicate other features discussed in the text 
Figure 8. Velocity amplitude profiles vs radius presented in Figure 6 for vortices NPC and SPC are compared against power law profiles $v=\mathrm{c} \mathrm{r}^{-\gamma}$. Panel a: NPC. $C_{1}$ is such to provide a speed of $65 \mathrm{~m} / \mathrm{s}$ at $1500 \mathrm{~km}$ from estimated vortex centre. Panel b: SPC. $C_{1}$ is such to provide a speed of $70 \mathrm{~m} / \mathrm{s}$ at $2000 \mathrm{~km}$ from estimated vortex centre. 

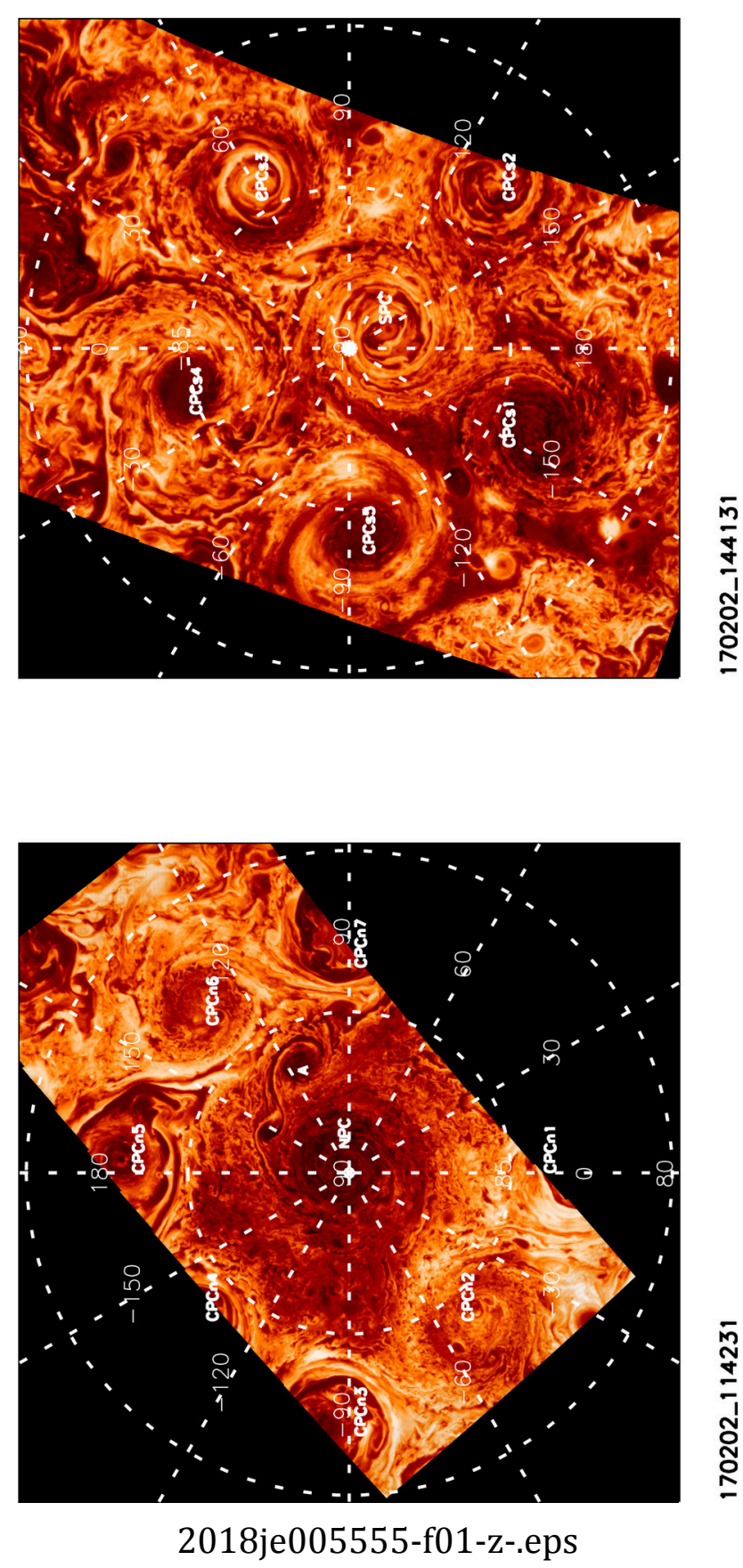

This article is protected by copyright. All rights reserved. 

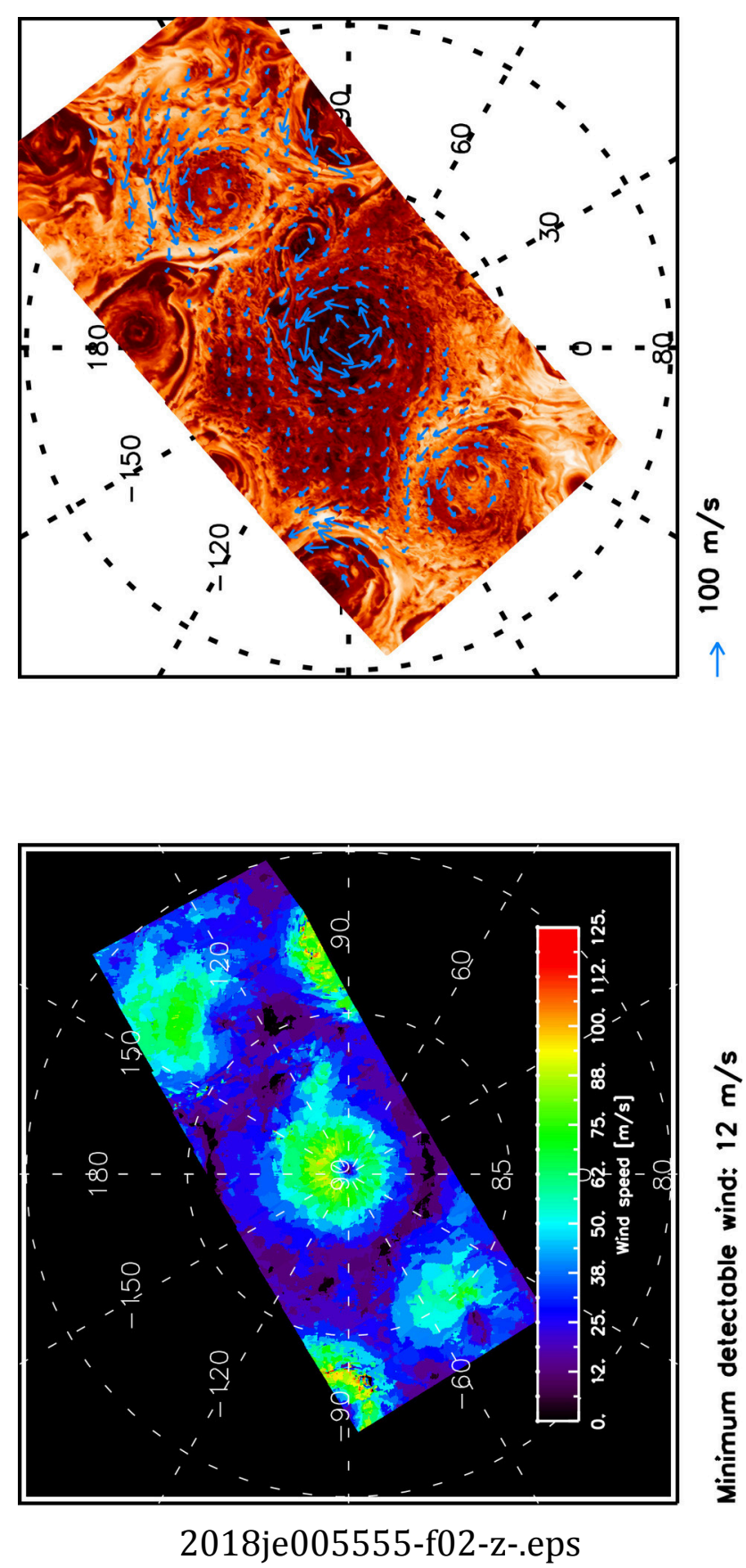

This article is protected by copyright. All rights reserved. 


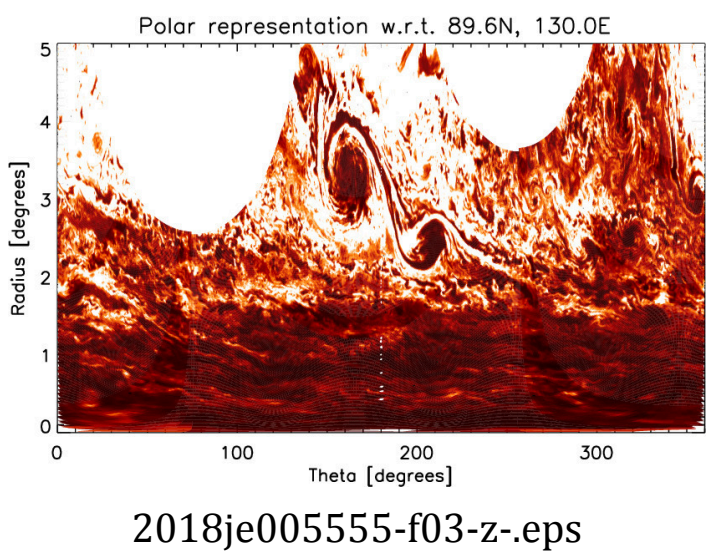

This article is protected by copyright. All rights reserved. 

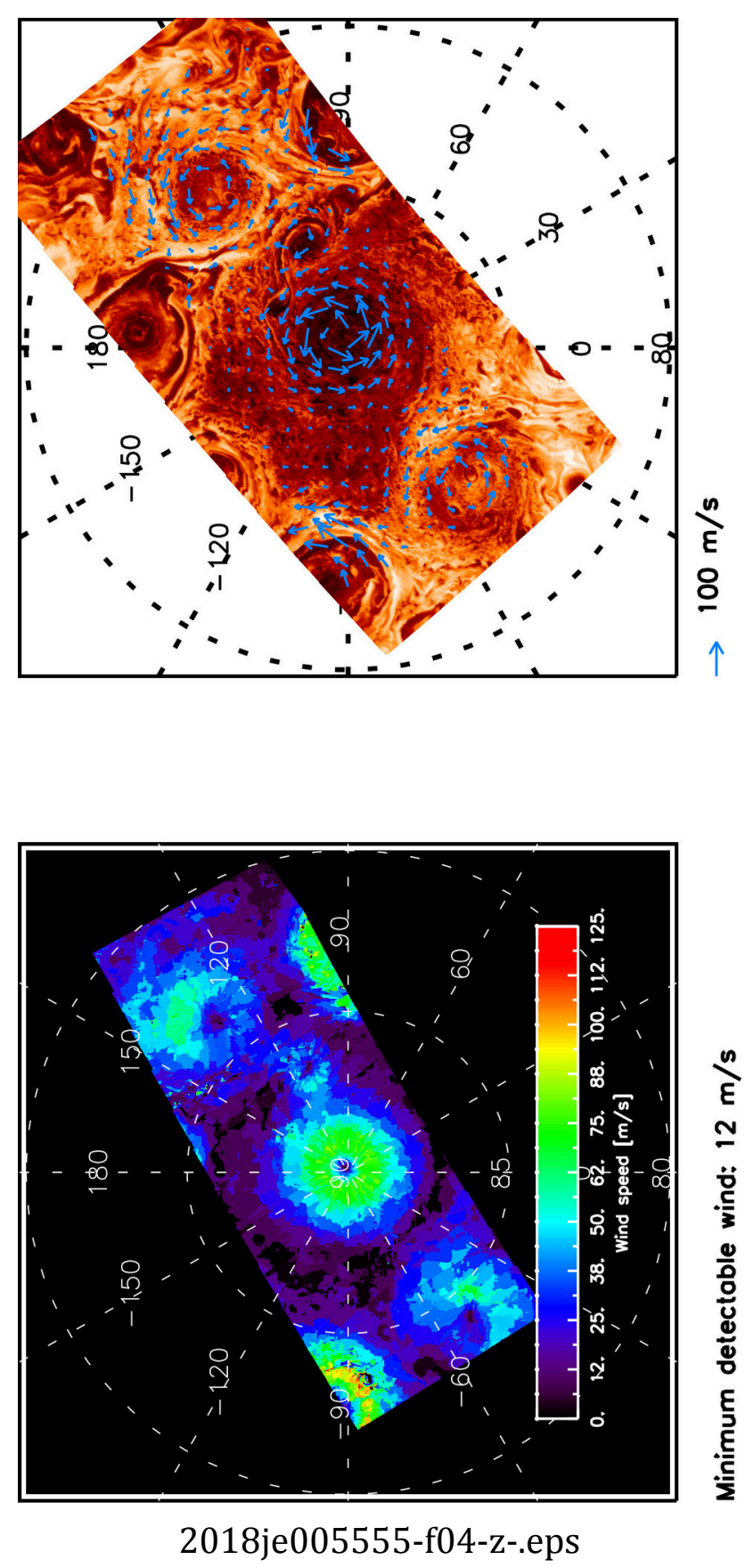

This article is protected by copyright. All rights reserved. 

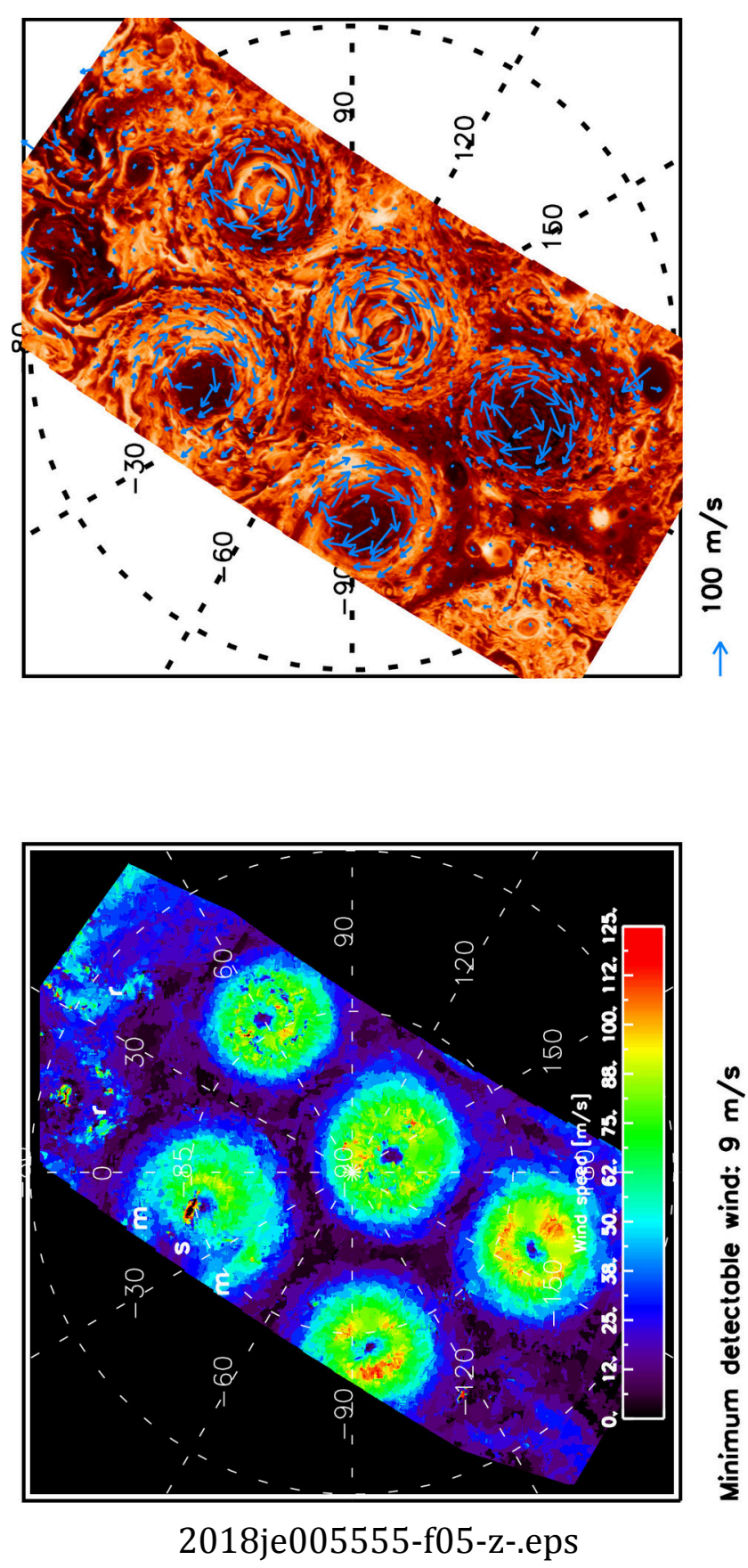

This article is protected by copyright. All rights reserved. 

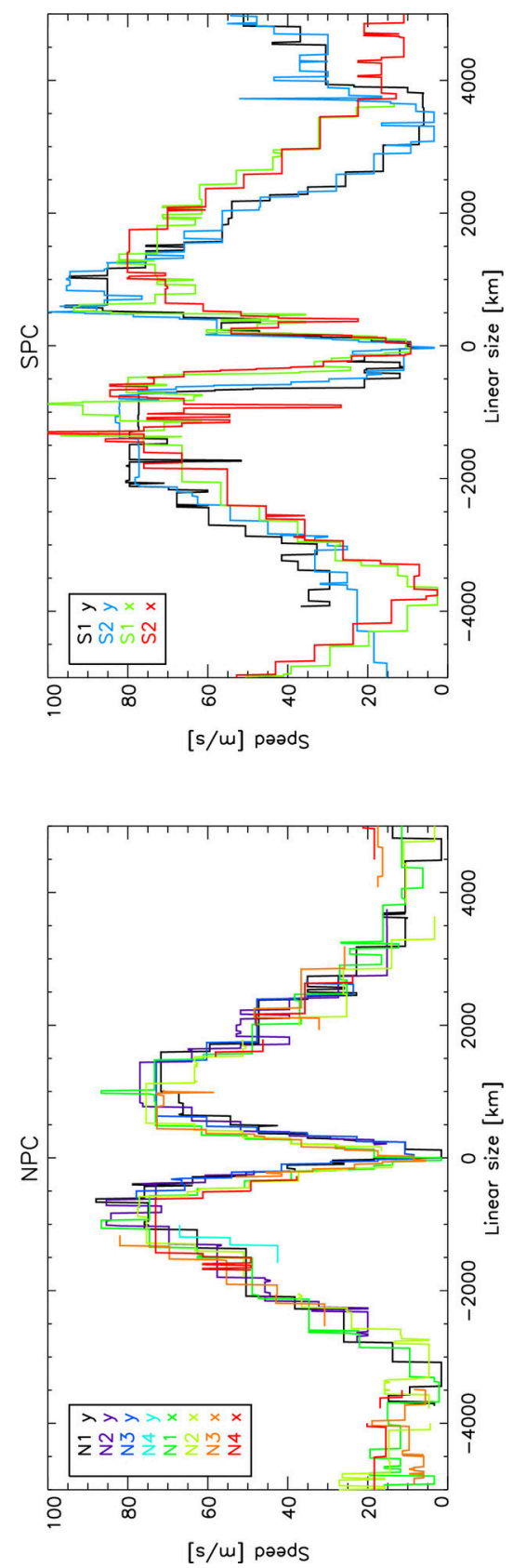

2018je005555-f06-z-.eps

This article is protected by copyright. All rights reserved. 

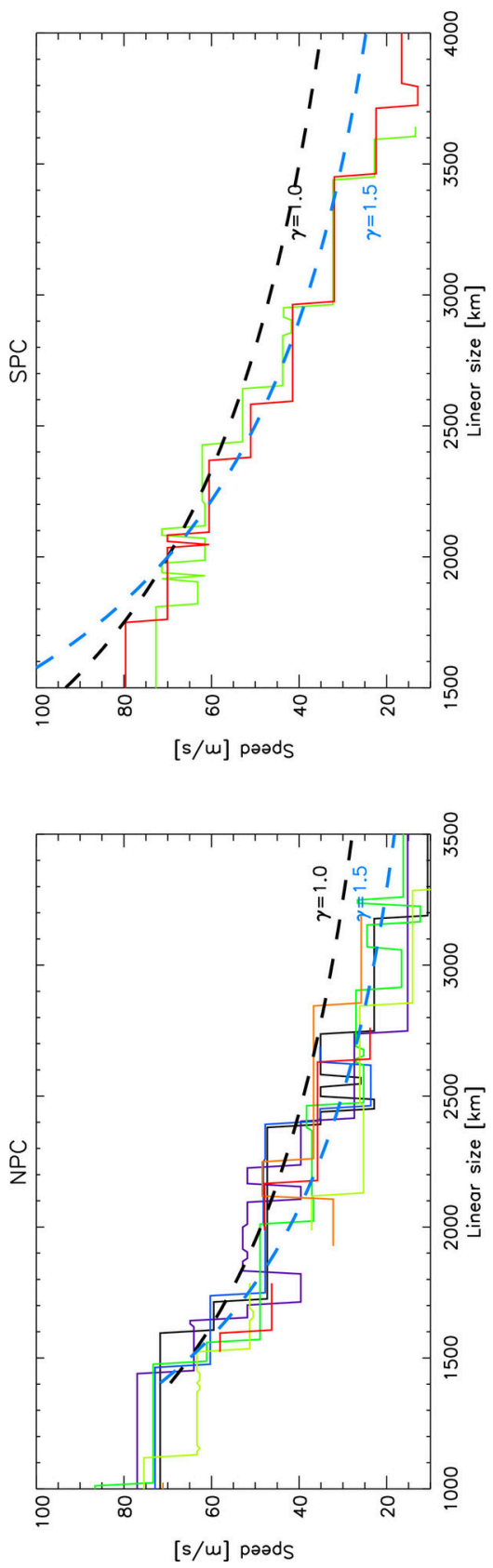

2018je005555-f08-z-.eps

This article is protected by copyright. All rights reserved. 